\title{
Reproductive Parameters and Characteristics of
}

\section{Labrador Breed}

\author{
Carlos Sorribas ${ }^{1}$, Mónica Pirles ${ }^{1}$, Laura Schiaffino ${ }^{1}$ and Carlos Pagni ${ }^{2}$ \\ 1. Clinic of pets. National University of Rosario. Ov Lagos and Route 33. 2170, Casilda. Argentinian Republic \\ 2. English. National University of Rosario. Ov Lagos and Route 33. 2170, Casilda. Argentinian Republic
}

\begin{abstract}
For a period of two years, a survey of 110 parturitions of Labrador breed bitches was carried out in Rosario City to determine the average number of puppies per litter, percentage of dystocia of all parturitions, percentage of males and females born and causes of dystocia, as well as the incidence of clinically detectable congenital pathologies. Over a total of 110 parturitions ninety three (84.5\%) parturitions were normal and 17 (15.5\%) were dystocic ones, it was established that atony 1 was the most numerous cause of dystocia (53\%), followed by atony 2 (23\%), dystocia by fetal disorders (6\%), hydramnios (6\%), uterine rupture (6\%) and vagina fibrous band (6\%). The whole puppies born was 866, 7.9 puppies per litter, if comparison between normal parturitions and dystocic ones is made, numbers are 7.05 and 8.02 respectively. When sex evaluation percentage is made $51.5 \%$ are males and $48.5 \%$ are females. When viability of puppies born is calculated it is established that in ones born by normal parturition perinatal death was $9.3 \%$, while in those born by dystocia it was $10.9 \%$. Among clinically verifiable congenital disorders low weight at birth was the most frequent congenital alteration, followed by cleft palate, lethal congenital edema or walrus syndrome and hare lip.
\end{abstract}

Key words: Labrador retriever, parturition, bitches, dystocia, congenital, hydramnios.

\section{Introduction}

Today, because of society emphasis on deliberated breeding of purebred animals, the veterinarian faces relative problems to have a successful parturition in these animals. Nowadays, the breeder in association with the veterinarian uses planned breeding methods to achieve a high conception rate, pregnancy and puppy survival to overcome published parameters [1].

The emotional values that dogs have in society, in addition to the interest with which breeders perform race selection make reproduction compromised in determined specific dog races or any specific animal, hence there is the need of knowing and promoting different options to improve birth rates and to decrease morbidity and mortality existing today in canine parturitions [1].

Management and treatment of dystocia is an important parameter to take into account and to

Corresponding author: Carlos Sorribas, Ph D. Associate professor, research fields: small animal reproduction. improve the performance of breeding places [2].

Labrador breed is not one of the most complicated at parturition moment, they were communicated [3] the ten most complicated races at birth moment: Boston terrier, English Bulldog, French Bulldog, Mastiff, Scottish Terrier, Miniature Bull Terrier, Drahthar, Clumber Spaniel, Pekinese and Dandie Dinmont Terrier.

\section{Materials and Methods}

The general aim of this work is to know in a detailed form the development of canine parturition and specifically on Labrador Retriever breed, to achieve this general aim a number of partial objectives will be developed and analyzed:

- To evaluate the incidence of dystocia in the breed.

- To evaluate causes of dystocic parturitions.

- To evaluate bitch age on the parturition development and on the litter size.

- To evaluate the importance of gestation and 
parturition tracing on the final result, number of born alive, normal and viable puppies per litter.

- To evaluate the percentage of male and female born.

- To compare litter size born by normal and dystocic parturition as well as the viability of puppies per breed.

- To determine the prevalence of principal congenital diagnosable diseases at birth by breed and by ages.

- To compare the number of born alive and viable puppies among those born by eutocic and dystocic parturitions.

A whole of 110 parturitions of Labrador Retriever bitches were studied, being them healthy with a complete health plan, all of them fed with a first-quality balanced food adapted to the gestational time. Puppies born were studied at birth and during the first 72 hours of life.

Results obtained were passed to an Excel sheet in order to ease the subsequent statistical study.

\section{Results}

From the whole of parturitions studied 866 puppies born were registered making an average of 7.9 puppies per litter.

Prevalence of dystocic parturitions in the breed is of $15.5 \%$, Table 1 [4] informed that dystocia frequency in Boston Terriers was $62 \%$ and that $43 \%$ of French
Bulldog breed parturitions was dystocic. In 2009 Bergström published in his doctoral thesis an incidence of 5.7 per thousands of parturition disorders in the studied population [5], communicated an incidence of 32\% in Boxer breed and 27.7\% in canine species, Boston Terriers [3] presented 92\% of dystocic parturitions, in dogo Argentine breed $89.3 \%$ of eutocic parturitions parturitions and $10.7 \%$ of dystocic [6].

Primary uterine inertia is the most frequent cause of dystocia in Labrador breed, in Table 2, according to this survey, agreeing with the communication [7] and is caused by an increase in the level of serum progesterone at the end of the gestation and a decrease of oxitocin and vasopresin, determining the levels of these hormones in the development of the primary atony or primary uterine inertia during the parturition process [8]. Several authors have communicated the most predisposed breeds to develop dystocia by primary uterine inertia or primary atony: Labrador, Italian Greyhound, Boxer, Mastiff, Great Danish, Mastiff of the Pyrinees, brachycephalic, Scottish Terriers and Dachsund [9-11].

The average number of pups born is 7.9 pups per litter, if the comparison is made between births of normal births 7.05 and dystocic births 8.02. Fig. 1 allows us to infer that the size of the litter is a factor predisposing for the development of the primary cause of dystocia, primary uterine inertia.

With respect to sex of puppies born it can be seen

Table 1 Percentage of normal parturitions or eutocic ones or difficult parturitions or dystocic ones.

\begin{tabular}{lll}
\hline Normal parturition & 93 & $84.5 \%$ \\
\hline Dystcic Parturition & 17 & $15.5 \%$ \\
Total & 110 & $100 \%$ \\
\hline
\end{tabular}

Table 2 Number and percentage of registered dystocia.

\begin{tabular}{lll}
\hline Type of dystocia & Number & Percentage \\
\hline Atony 1 & 9 & $53 \%$ \\
Atony 2 & 4 & $23 \%$ \\
Fetal Alterations & 1 & $6 \%$ \\
Hydramnios & 1 & $6 \%$ \\
Uterine Rupture & 1 & $6 \%$ \\
Vagina Fibrous Band & 1 & $6 \%$ \\
Total & 17 & $100 \%$ \\
\hline
\end{tabular}




\section{Average number of pups born}

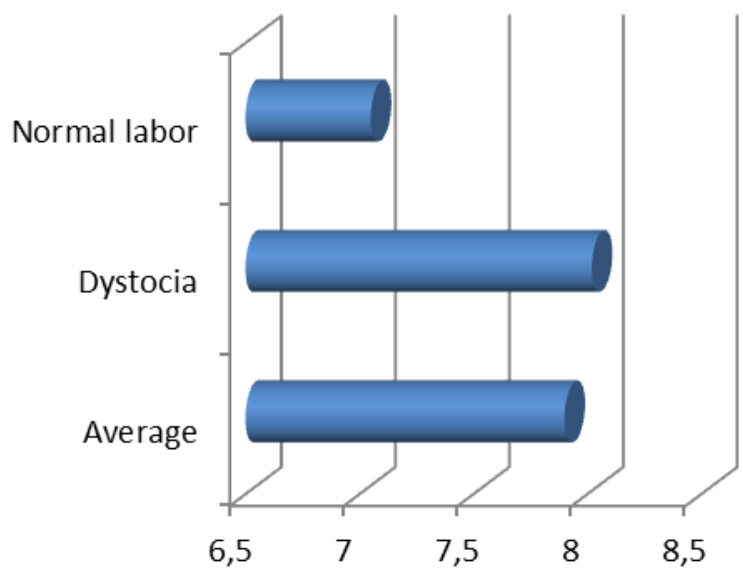

Average number of pups born

Fig. 1 Average of puppies born by type of parturition compared to general average.

\section{Percentage of males and females born}

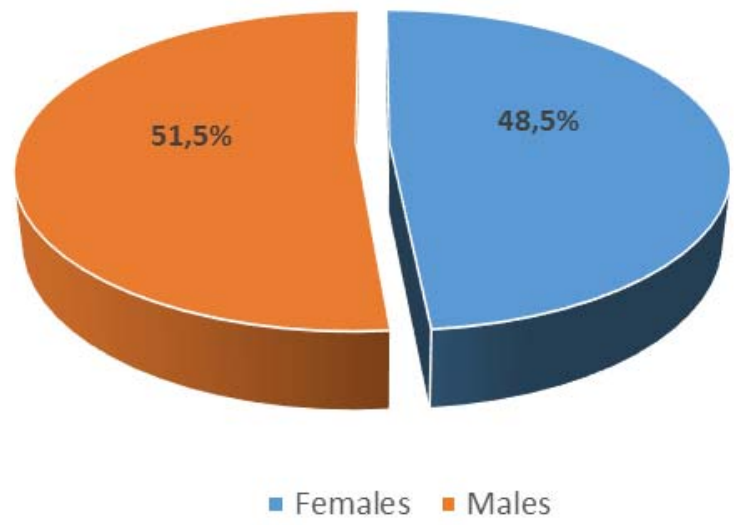

Fig. 2 Percentage of puppies born by sex.

that 1.08 males were born per female born, in Fig. 2, unlike what is communicated [12], a percentage of 1.25 females per male born, but it must be considered that it is based on different populations, while the present work is based on a particular breed, the other one is based on canines in general.

Perinatal death appears from the last week of pregnancy to the first 72 hours after birth, in this work it could be found a percentage of $9.3 \%$ of perinatal mortality in puppies born by natural or eutocic parturition and $10.9 \%$ in those born by dystocia.

\section{Conclusions}

Labrador retriever is a breed having little parturition difficulty with only $15.5 \%$ of dystocic parturitions. Average of puppies born per litter is 7.9. The most common dystocia in the breed is caused by primary uterine inertia or primary atony, dystocia from maternal causes far exceed those provoked by fetal causes. It is observed a higher growth of male birth, 1.08 males per female born. Breed perinatal mortality is $10.1 \%$, perinatal mortality by dystocic parturition is 
$10.9 \%$ and $9.3 \%$ by normal or eutocic parturitions. Low weight at birth was the most frequent congenital alteration among the clinically verifiable congenital disorders, followed by cleft palate, lethal congenital edema or Walrus Syndrome and harelip.

\section{References}

[1] Smith, F. O. 2007. "Challenges in Small Animal Parturition-Timing Elective and Emergency Cesarean Sections.” Theriogenology 68: 348-53.

[2] Munnich, A., and Kuchenmeister, U. 2009. "Dystocia in Numbers-Evidence-Based Parameters for Intervention in the Dog: Causes for Dystocia and Treatment Recommendations.” Reprod Domest Anim 44 (Suppl. 2): 141-7.

[3] Evans, K. M., and Adams, V. J. 2010. "Proportion of Litters of Purebred Dogs Born by Caesarean Section.” $J$ Small Anim Pract. 51 (2): 113-8. doi: 10.1111/j.1748-5827.2009.00902.x.

[4] Eneroth, A, Uhlhorn, M., Swensson, L., Linde-Forsberg, C., and Hall, M. 2000. "Valpnings Problem Hos Fransk Bulldog, Skotsk Terrier \& Boston Terrier (Dystocia in the French Bulldog, Scottish terrier and Boston terrier).” Hundsport 112 (9): 38-41.

[5] Linde Forsberg, C., and Persson, G. 2007. "A Survey of Dystocia in the Boxer Breed.” Acta Vet Scand. 49: 8.
[6] Caffaratti, M., Gonzalez, G., Gorla, N., and Guendulain, C. 2012. "Reproductive Parameters of Dogo Argentino bitch." In Proceedings of the 7th International Symposium on Canine and Feline Reproduction, ISCFR, 2012, Whistler, Canada.

[7] Davidson AP. Primary uterine inertia in four labrador bitches J Am Anim Hosp Assoc. 2011 Mar-Apr;47(2):83-8. doi: 10.5326/JAAHA-MS-5122. Epub 2011 Feb 10.

[8] Bergström, A., Fransson, B,, Lagerstedt, A., and Kindahl, H. 2010. "Hormonal Concentrations in Bitches with Primary Uterine Inertia.” Theriogenology 73 (8): 1069-75.

[9] Johnston, S, Root Kustritz, M., and Olsen, P. 2011. “Canine Parturition Eutocia and Dystocia." In Canine and Feline Theriogenology, edited by Kersey R., Le Melledo D. Saunders, Philadelphia, pp. 105-28.

[10] Buff, S. 2002. "Mise bas et dystocie chez le chien et le chat. Nouv. Prat. Vét.” In hors série Hospitalisation, pp. 117-20.

[11] Lennoz-Roland, M. 2008. Conduite à tenirdevantune dystocie chez la chienne. Le Point Vétérinaire, 39 (numéro spécial: Actualitésen reproduction du chien et du chat), pp. 43-9.

[12] Ajala Oluwatoyin, O., Fayemi, O. E., and Oyeyemi, M. O. 2012. "Some Reproductive Indices of the Nigerian Local Bitches in Ibadan, Nigeria.” Niger J Physiol Sci 27 (1): 49-53. 